\title{
Review of: "Risk factor-based optimal endoscopic surveillance intervals after endoscopic submucosal dissection for gastric adenoma"
}

masaichi Ohira

Potential competing interests: The author(s) declared that no potential competing interests exist.

Thank you for giving me the opportunity to review this interesting manuscript titled, " Risk factor-based optimal endoscopic surveillance intervals after endoscopic submucosal dissection for gastric adenoma ". The authors proposed a simple and promising strategy for determining a better endoscopic surveillance interval by parameterizing diverse and group-specific recurrence risk factors into a well-known survival model, which is well understandable and is able to be satisfied. I have a couple of questions.

1. What is the true character of protruded scar? Is it neoplasm or granulation? If the author found protruded scar 3 months after ESD, How will you do ? Will you follow or will you take biopsy specimen?

2. In baseline characteristics of Table1 pathological results of differentiation of the resected adenoma, that is low grade dysplasia or high grade dysplasia were not described. Was the frequency of recurrence different between these two types of adenoma?

3. In Discussion line15-16, the same sentence was described " There are few studies on scar morphology. 